\title{
Sensitivity of the Cherenkov Telescope Array to Dark Subhalos
}

\section{Javier Coronado-Blázquez, ${ }^{a, b, *}$ Michele Doro, ${ }^{c}$ Miguel A. Sánchez-Conde ${ }^{a, b}$ and Alejandra Aguirre-Santaella ${ }^{a, b}$}

${ }^{a}$ Instituto de Física Teórica IFT UAM-CSIC, C/ Nicolás Cabrera 13-15, Madrid, Spain

${ }^{b}$ Departamento de Física Teórica, Universidad Autónoma de Madrid, Campus de Cantoblanco, Madrid, Spain

${ }^{c}$ Dipartimento di Fisica e Astronomia Galileo Galilei of Università degli Studi di Padova \& INFN Padova, I-35131 Padova, Italy E-mail: javier.coronado@uam.es, michele.doro@unipd.it, miguel.sanchezconde@uam.es, alejandra.aguirre@uam.es

In this work, we study the potential of the Cherenkov Telescope Array (CTA) for the detection of Galactic dark matter (DM) subhalos. We focus on low-mass subhalos that do not host any baryonic content and therefore lack any multiwavelength counterpart. If the DM is made of weakly interacting massive particles (WIMPs), these dark subhalos may thus appear in the gamma-ray sky as unidentified sources. A detailed characterization of the instrumental response of CTA to dark subhalos is performed, for which we use the ctools analysis software and simulate CTA observations under different array configurations and pointing strategies, such as the scheduled extragalactic survey. This, together with information on the subhalo population as inferred from N-body cosmological simulations, allows us to predict the CTA detectability of dark subhalos, i.e., the expected number of subhalos in each of the considered observational scenarios. In the absence of detection, for each observation strategy we set competitive limits to the annihilation cross section as a function of the DM particle mass, that are at the level of $\langle\sigma v\rangle \sim 4 \times 10^{-24}$ $\left(7 \times 10^{-25}\right) \mathrm{cm}^{3} \mathrm{~s}^{-1}$ for the $b \bar{b}\left(\tau^{+} \tau^{-}\right)$annihilation channel in the best case scenario. Interestingly, we find the latter to be reached with no dedicated observations, as we obtain the best limits by just accumulating exposure time from all scheduled CTA programs and pointings over the first 10 years of operation. This way CTA will offer the most constraining limits from subhalo searches in the intermediate range between $\sim 1-3 \mathrm{TeV}$, complementing previous results with Fermi-LAT and HAWC at lower and higher energies, respectively.

$37^{\text {th }}$ International Cosmic Ray Conference (ICRC 2021)

July 12th - 23rd, 2021

Online - Berlin, Germany

\footnotetext{
*Presenter
} 


\section{Introduction}

Ground-based very-high-energy (VHE) gamma-ray astronomy has been richly developed in the last decades. Several Imaging Atmospheric Cherenkov Telescopes (IACTs) are currently in operation, namely H.E.S.S., MAGIC, and VERITAS. IACT observations have been used to place competitive limits on $\mathrm{TeV}$-scale dark matter (DM) candidates, although still unable to probe the theoretically motivated annihilation thermal relic cross section value [1].

The Cherenkov Telescope Array (CTA) [2] is the next-generation ground-based gamma-ray observatory. Currently under construction phase, it will consist of two arrays: a Southern one located at the ESO site in the Atacama desert, Chile, and a Northern one located at the Roque de los Muchachos in La Palma, Canary Islands, Spain. These two arrays, in both hemispheres, will make CTA the first ground-based gamma-ray telescope able to observe almost the whole sky.

As a result of this setup, CTA will cover energies ranging from a few $\mathrm{GeV}$ up to hundreds of TeV. The large number of telescopes will also enable CTA to perform, for the first time, large surveys of extended sky regions at the highest energies. Indeed, an extragalactic survey with CTA is one of the so-called key science projects (KSPs). These represent the core topics within the CTA science program to be addressed over the first years of operations. In addition to VHE astrophysics and cosmology, these KSPs include a total of nearly 1,500 hours devoted to the search of DM [2]. Especially relevant in this context will be the dedicated search for annihilating DM with CTA at the Galactic Center, amply discussed in Ref. [3]. We must stress out that the detailed specifics of CTA KSPs are still a matter of debate, and may be refined before CTA actually starts observations.

In addition to the already "traditional" astrophysical targets to search for DM annihilation (e.g., Galactic center, dwarf galaxies), there is the possibility to use CTA for less explored DM scenarios such as the one offered by the so-called Galactic dark subhalos. These represent the less massive components of DM halo substructures in our galaxy, not massive enough to retain a baryonic, visible counterpart, as opposed to the case of the larger subhalos that host dwarf galaxy satellites. Being invisible in most of the electromagnetic spectrum, dark subhalos are hard to locate. Yet, despite their unknown location, they can be excellent candidates for gamma-ray DM searches given their typical number densities, masses and distances [4]. Indeed, several groups have searched for dark subhalos in gamma-ray data and catalogs, and were able to set competitive constraints in the absence of them [5, 6]. For this work we identify three different scenarios in which a subhalo may appear in the CTA FoV:

EGAL scenario: the CTA planned Extragalactic Survey. CTA plans to perform the largest sky survey ever performed by IACTs, covering a fraction of $25 \%$ of the extragalactic $\left(b>5^{\circ}\right)$ sky with an uniform exposure of $3 \mathrm{~h}$ per pointing and a total of $1000 \mathrm{~h} \mathrm{[2].}$

DEEP Scenario: a deep exposure over a specific sky area. CTA will be operated as an open observatory with a guest observation program. Possible requests could be aimed at deep wide-field sky observation, in which a specific sky region is observed for prolonged time ( $100-150$ hours). Thanks to divergent pointing mode, CTA can scan a region of sky as narrow as about $8 \times 8 \mathrm{deg}^{2}$ or as large as $20 \times 20 \mathrm{deg}^{2}$ without need of repointing.

EXPO scenario: overall CTA 10 years exposure. After 10 years of continous operations, CTA is expected to have covered a considerable fraction of the sky as overall sum of its individual 
pointings. One could combine all individual exposures and argue that potential dark subhalos could appear as serendipitous sources within the FoV of such pointings and exposures.

The goal of this paper is to evaluate the sensitivity of the CTA to dark subhalos for each of these different observational strategies. We will also provide constraints on the annihilating dark matter $\langle\sigma v\rangle-m_{\chi}$ parameter space in case none of these observing scenarios would provide a detection.

\section{Dark subhalo search scenarios}

Unless directly spotted by gravitational probes or detected in gamma-rays by wide FoV instruments in satellites (e.g. Fermi-LAT) or atmospheric shower ground-based particle detectors (e.g., HAWC), dark subhalos can be found only serendipitously in the CTA FoV. This can be attained in scenarios that provide large sky areas or exposures: an extragalactic sky scan, a deep wide-field over a certain sky region or as excess signals within the FoV of regular operation observations. In the following, we discuss in more details the putative detection scenarios identified above.

\subsection{EGAL}

The EGAL survey is a CTA KSP by the CTA Consortium, as it will offer an unprecedented, unbiased view of the TeV extragalactic sky [2]. The current plan is to scan $25 \%$ of the sky with an uniform exposure of $3 \mathrm{~h}$ per pointing, for a total of $1000 \mathrm{~h}$ over 3 years.

The main advantage of this search strategy is having access to a wide area of the sky surveyed with homogeneous acceptance, thus allowing for unbiased results. Furthermore, being a CTA KSP, its execution is guaranteed, although the exact sky region is not fixed yet. It is then possible that within this wide area a few DM subhalos will be present. A drawback of this observing strategy is the relatively short exposure per pointing, with a reported average sensitivity of the full survey of around $6 \mathrm{mCrab}\left(\sim 3.04 \times 10^{-13} \mathrm{ph} \mathrm{cm}^{-2} \mathrm{~s}^{-1}\right)$ for an energy threshold of $125 \mathrm{GeV}$.

\subsection{DEEP}

A dedicated, long exposure of $100-200 \mathrm{~h}$ on a specific sky region of extended FoV between $8 \times 8 \mathrm{deg}^{2}$ and $20 \times 20 \mathrm{deg}^{2}$ appears as a possible observation mode in CTA and consequently a viable scenario for dark subhalo searches with CTA as well. Deep-field observations can be proposed in the future to CTA either by Consortium members or via the CTA guest observational program. Such regions could be observed by CTA in focused, patched observation, or divergent pointing mode, in which the FoV is enlarged by slightly misaligning the telescopes within the array.

Dark subhalos could serendipitously fall within the FoV, whereas the probability of detection depends on the chosen sky region and its area. As a benchmark model, we will adopt a deep wide-field of $10 \times 10 \mathrm{deg}^{2}$ with $100 \mathrm{~h}$ of exposure. Note though that different sky regions, different extensions, and different exposures could be required for specific science cases.

The main drawback of this mode is that the surveyed area is relatively small, and therefore the probability to spot a dark subhalo by chance is low. Furthermore, deep, long-duration observations may be subject to higher systematics related, e.g., to the background fluctuations, inevitable over large period of times, considering the background rate depends both on the position in the sky and the atmospheric conditions during a specific data-taking. Such systematics effects are not taken into account in our estimates below. 


\subsection{EXPO}

During its lifetime, CTA will be accumulating exposures on several directions of the sky, observation by observation. It is thus certainly possible that a dark subhalo may serendipitously appear within one of these sky regions. While it is not possible to predict exactly the overall CTA exposure and its sky footprint, one can make educated guesses based on available data from currently operating IACTs.

We base our calculation of the total, integrated CTA observation area/time as obtained after its first 10 years of data forecasting it from the actual operations of MAGIC, an IACT currently in operation [7]. MAGIC is at the same place of the northern CTA array. The MAGIC overall exposure map allows us to predict a plausible exposure map for CTA. We extrapolated MAGIC observations in stereo configuration from November 2012 to June 2019, i.e., 6.5 years of data into a projected exposure map for CTA for 10 years of operation. Our results are shown in Figure 1. The total CTA predicted observed area is roughly $45 \%$ of the sky - almost twice that of the EGAL survey footprint, yet unevenly sampled in terms of exposure time.
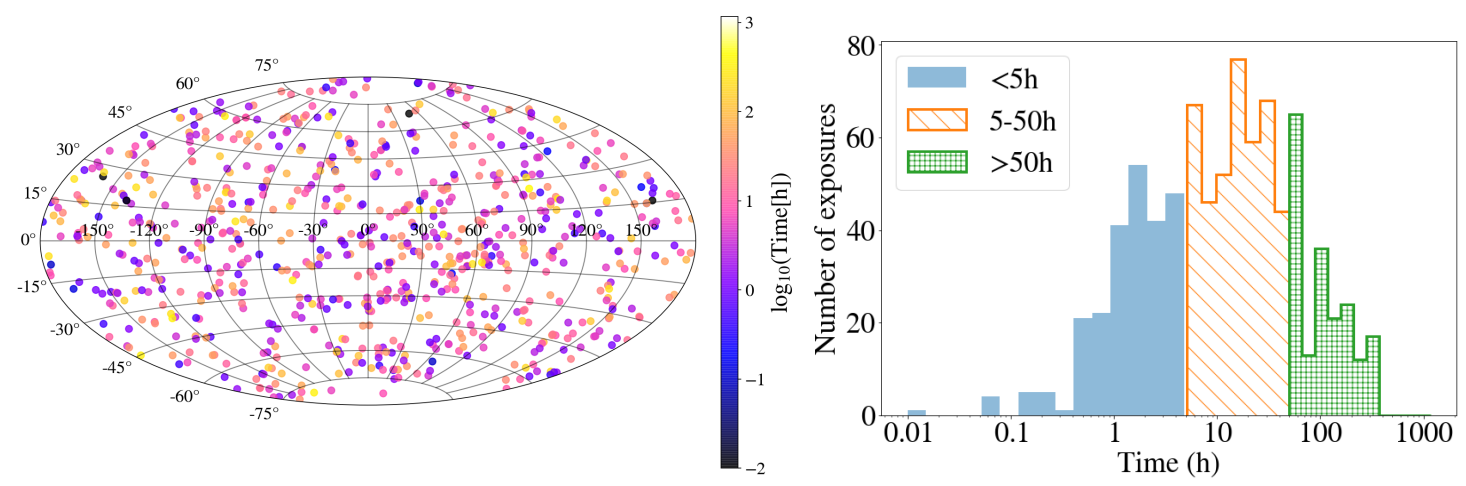

Figure 1: Left panel: overall 10-year exposure map for CTA in Galactic coordinates, extrapolated from 6.5 years of MAGIC stereo observations. The shown map corresponds to a random realization of our extrapolation algorithm. Color traces the observation time per pointing. Right panel: distribution of the observation time of the EXPO strategy for three bins: 0-5h, 5-50h and $>50 \mathrm{~h}$, for the same random realization.

The right panel of Figure 1 shows the distribution of exposure times for all the simulated CTA pointings in a random realization, where $\sim 33 \%$ of the total exposure time is devoted to short $(<5)$ h observations, $\sim 47 \%$ of $(5-50)$ h duration dominate the observational program, and $\sim 20 \%$ observation time is spent for very deep $(>50)$ h observations. For a fixed survey area, chances of serendipitous detection of dark subhalos are maximized during long or very long observations, as they will allow detection of DM candidates with small annihilation cross sections. Note that, in case of no-detection, DM limits can still be computed by using the full exposure map available at the time (Section 4).

\section{Dark subhalo detectability}

In this section, we evaluate the different performance of CTA to specific DM annihilation spectra. Indeed, the CTA sensitivity to DM-induced signals is expected to be different that the 
one for more common astrophysical "featureless" power-law spectra. Thus, the computation of this CTA DM sensitivity is critical to properly address the actual chances of dark subhalo discovery.

For instance, for the EGAL survey, an integral sensitivity of $\sim 6 \mathrm{mCrab}$ is quoted. Yet, this sensitivity was computed for a Crab Nebula-like spectrum, that can be well approximated by a single power-law with a spectral index -2.62 . Instead, DM annihilations show more complex, highly-curved spectra, strongly dependent on the mass of the WIMP and specifics of the considered annihilation channel, and with a more or less sharp cutoff at the DM mass.

To correctly compute the CTA sensitivity to DM spectra, we use the public ctools ${ }^{1}$ software [8], v1.7. We compute the sensitivity of CTA to dark subhalos in the EGAL scenario by placing a simulated point-like subhalo -made of WIMPs of a given mass and annihilating via a particular channel- in a position of the Northern Galactic hemisphere covered by the EGAL scan and close to the zenith angle of the instrumental response function (IRF) we use (i.e., $40^{\circ}$ ). We adopt 3 hours per pointing so as to match the EGAL survey setup reported in Ref. [2].

Individual events with energies between $30 \mathrm{GeV}$ up to $100 \mathrm{TeV}$ are generated for a particular DM annihilation channel and WIMP mass by means of the ctobssim Monte Carlo generator. The input is a spectral file function obtained from PPPC4DMID [9], generated with PYTHIA 8 and including electroweak corrections. Only $b \bar{b}$ and $\tau^{+} \tau^{-}$annihilation channels are considered in this work as representative of "soft" and "hard" DM spectra, respectively, i.e., spectra exhibiting shallower and steeper cutoffs at the DM mass.

The events are generated using the latest IRFs, prod3b-v2, with the North_z40_5h ${ }^{2}$ (i.e., CTA Northern array, for a source located at a zenith angle of $40^{\circ}$, azimuth-averaged for $5 \mathrm{~h}$ observation time). Once the events are simulated, we use ctlike to compute the detection significance via the likelihood-ratio test statistic (TS), defined as:

$$
\mathrm{TS}=-2 \log \left[\frac{\mathcal{L}\left(H_{1}\right)}{\mathcal{L}\left(H_{0}\right)}\right]
$$

where $\mathcal{L}\left(H_{0}\right)$ and $\mathcal{L}\left(H_{1}\right)$ are, respectively, the likelihood functions under the null (no source) and alternative (existing source) hypotheses. The detection threshold is set to $\mathrm{TS}=25$, corresponding to about 5 standard deviations [10]. For each mass and annihilation channel, the normalization of the source flux is varied by running ctlike iteratively until the detection threshold is reached. We adopt a tolerance of $T S=25 \pm 1$ to ensure numerical convergence. The flux obtained this way is the minimum detection flux, $F_{\min }$.

The results of our DM sensitivity computation are shown in Figure 2 for the $b \bar{b}$ and $\tau^{+} \tau^{-}$ annihilation channels. Both channels present similar behaviours. Standard Crab-based integral sensitivity overestimates the actual DM performance by a factor of $10-100$ for low DM mass (depending on the specific channel), while the sensitivity to DM spectra reaches the standard one toward larger masses, to become even better (by a factor of up to 10 at the heaviest considered WIMP) in the case of the $\tau^{+} \tau^{-}$channel.

We now elucidate the number and the flux of expected dark subhalos for each specific sky region of the three scenarios described in Section 2. To perform this task, we choose the procedure to populate N-body simulations with low-mass dark clumps as described by [5, 6], which are based

${ }^{1}$ http://cta.irap.omp.eu/ctools/

${ }^{2}$ Publicly available at https://www.cta-observatory.org/science/cta-performance/ 

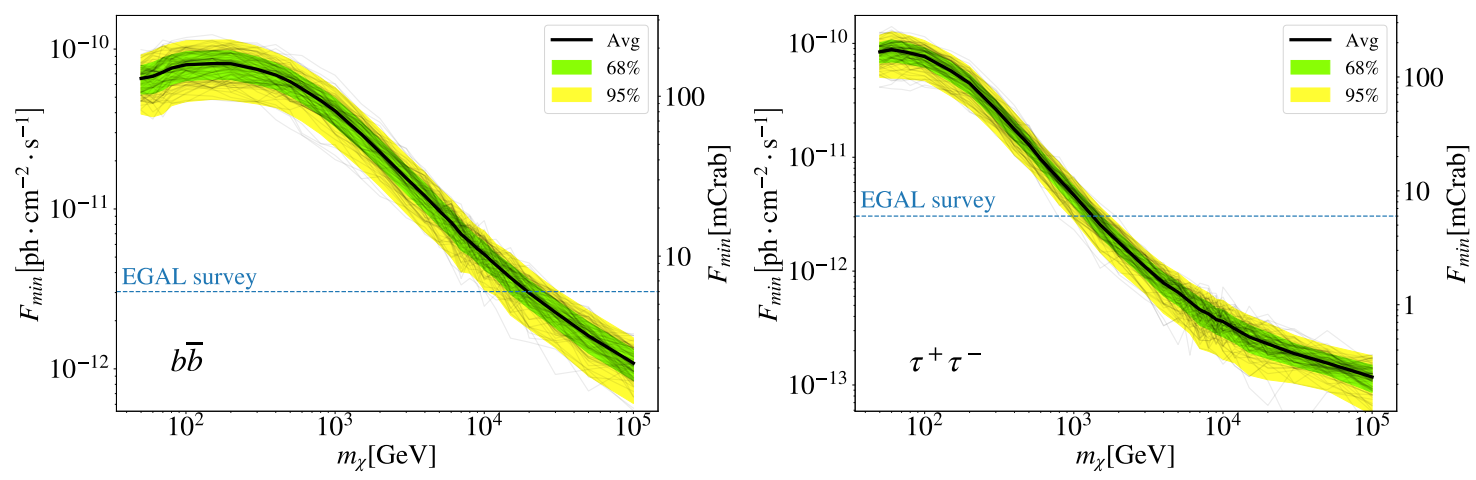

Figure 2: Minimum flux $\left(F_{\text {min }}\right)$ to detect a dark subhalo annihilating to $b \bar{b}$ (top panel) and $\tau^{+} \tau^{-}$(bottom panel) in the EGAL survey, assuming the Northern array and $3 \mathrm{~h}$ of normal pointing observation with an energy threshold of $30 \mathrm{GeV}$. Gray lines are the individual 100 realizations, black solid line is the average value, and green and yellow bands are, respectively, the $68 \%$ and $95 \%$ confidence level. Right $y$ axis is expressed in units of mCrab flux. Blue dashed, horizontal line is the nominal $6 \mathrm{mCrab}$ flux sensitivity of the EGAL scan.

on the Via Lactea II (VL-II) N-body cosmological simulation of a Milky Way-sized halo [4]. From the astrophysical point of view, the DM-induced "brightness" is codified into the so-called J-factor, which accounts for all the astrophysical considerations:

$$
J=\int_{\Delta \Omega} d \psi \int_{l . o . s} \rho_{D M}^{2}(r(l)) d l,
$$

where the first integral is performed along the sky line $\psi$ over the solid angle defined from the signal region $(\Delta \Omega)$, the second one along the line of sight (1.o.s, $l$ ), and $\rho_{D M}$ is the DM density profile of the object under consideration. In the following, we adopt for the J-factor the so-called $J_{\max }$ value, the one of the brightest subhalo, averaged across our realizations of VL-II in such a way that we take the value above which $95 \%$ of the brightest subhalo J-factor distribution is contained. $J_{\max }$ will also depend on the sky area considered, as explained below. The J-factor distribution of all realizations is drawn imposing $\mathrm{M} \leq 10^{8} \mathrm{M}_{\odot}$ as to ensure we deal with dark subhalos [11].

For a given total observation time, increasing the area will result in less time per pointing and a poorer sensitivity. Thus, the optimal strategy must rely on a compromise between area and time. For each of the three different observation strategies discussed in Section 2 it is now possible to estimate the number of expected subhalos in the CTA FoV above a certain J-factor, $J_{\text {min }}$. The results are summarized in Table 1, where the reported uncertainties are due to the variance averaging across realizations of the VL-II simulation and across different sky positions of the observed patch.

\section{DM constraints}

However, even if no dark subhalo is detected by CTA, it will be still possible to set constraints to the $\langle\sigma v\rangle-m_{\chi}$ DM parameter space. The methodology is the same as in Refs. [5, 6], and is based on a comparison between the $\mathrm{N}$-body simulation prediction $J_{\max }$ and the gamma-ray data $F_{\min }$.

Figure 3 shows the $95 \%$ c.l. upper limits to the DM annihlation cross section for the $b \bar{b}$ (top panel) and $\tau^{+} \tau^{-}$(bottom panel) annihilation channels for the three observational strategies under 


\begin{tabular}{c|c|c|c}
\hline & \multicolumn{3}{|c}{$N\left(\geq J_{\min }\right)$} \\
\hline $\log _{10}\left(J_{\min }\right)$ & EGAL & DEEP & EXPO \\
\hline 17 & $392 \pm 18$ & $26 \pm 8$ & $728 \pm 23$ \\
18 & $115 \pm 11$ & $5.1 \pm 2.5$ & $206 \pm 14$ \\
19 & $6.5 \pm 2.6$ & $0.3 \pm 0.5$ & $13 \pm 4$ \\
20 & $0.3 \pm 0.5$ & 0 & $0.5 \pm 0.7$ \\
\hline
\end{tabular}

Table 1: Average number of subhalos above a given $J_{\text {min }}$ in the CTA FoV for the three observation strategies of Section 2, across 1000 realizations of the repopulated VL-II N-body simulation.

consideration in this work. The most stringent limits are obtained for the EXPO method, while the weakest ones are those for which the DEEP scenario is adopted.
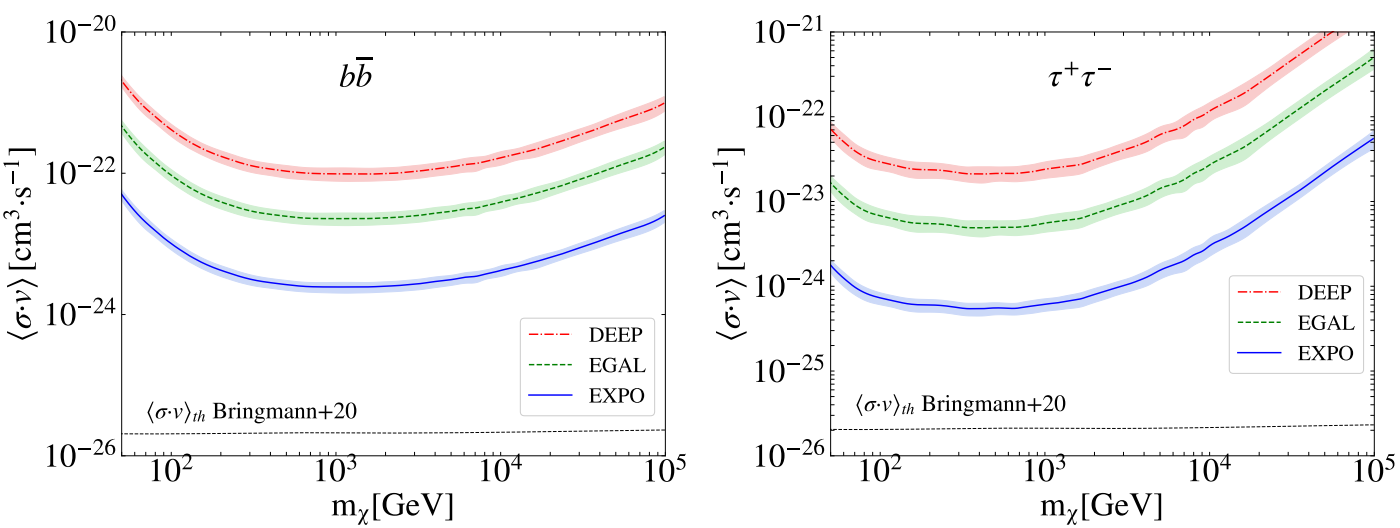

Figure 3: $95 \%$ C.L. upper limits to the DM annihilation cross section for $b \bar{b}$ (top) and $\tau^{+} \tau^{-}$(bottom) assuming no unIDs are detected by CTA under any of the three observational strategies proposed in Sec. 2: a dedicated $10 \times 10 \mathrm{deg}^{2}, 100 \mathrm{~h}$ deep-field, the EGAL survey, and the EXPO scenario.

These constraints reach their best sensitivity for masses of $\sim 1 \mathrm{TeV}(500 \mathrm{GeV})$ for the $b \bar{b}\left(\tau^{+} \tau^{-}\right)$ annihilation channel, of the order of $3 \times 10^{-24}\left(7 \times 10^{-25}\right) \mathrm{cm}^{3} \cdot \mathrm{s}^{-1}$. Interestingly, the behavior around the maximum sensitivity is fairly flat over more than an order of magnitude in DM mass, especially in the case of $b \bar{b}$. We recall that values roughly two orders of magnitude above the thermal relic cross section are ruled out for canonical WIMPs.

\section{Summary and conclusions}

In this paper, we assessed the detectability of dark subhalos with the Cherenkov Telescope Array (CTA). We proposed three different observational strategies based on the current Key Science Programs (KSPs) and the predictions for the sky pointings. A careful characterization of the sensitivity of CTA to dark subhalos in these observation scenarios was then performed by means of 100 simulations of a putative subhalo made of WIMPs annihilating via two different annihilation channels $\left(b \bar{b}\right.$ and $\left.\tau^{+} \tau^{-}\right)$.

Based on hundreds of realizations of the Via Lactea II N-body cosmological simulation, "repopulated" with subhalos well below the resolution limit of the original simulation, we computed the expected number of dark subhalos above a certain J-factor in the three different scenarios. 
Assuming instead that no dark subhalo is observed, we were able to compute the 95\% C.L. constraints to WIMP annihilation (Figure 3). This was done by combining CTA sensitivity results with predictions from our repopulated N-body simulations. The EXPO scenario offers the most competitive results, followed by EGAL and DEEP. The EXPO limits are of the order of $\langle\sigma v\rangle \sim 10^{-24}$ $\mathrm{cm}^{-3} \mathrm{~s}^{-1}$ for the $\tau^{+} \tau^{-}$channel and slightly above for the $b \bar{b}$ channel. Thus, they are comparable to those obtained with dwarf satellite galaxies by current IACTs.

\section{References}

[1] M. Doro, A decade of dark matter searches with ground-based Cherenkov telescopes, Nuclear Instruments and Methods in Physics Research Section A: Accelerators, Spectrometers, Detectors and Associated Equipment 742 (apr, 2014) 99-106.

[2] The Cherenkov Telescope Array Consortium, Science with the Cherenkov Telescope Array. World Scientific, 2017.

[3] The Cherenkov Telescope Array Consortium, Sensitivity of the Cherenkov Telescope Array to a dark matter signal from the Galactic centre, Journal of Cosmology and Astroparticle Physics 2021 (Jan, 2021) 057-057.

[4] J. Diemand, M. Kuhlen, P. Madau, M. Zemp, B. Moore, D. Potter et al., Clumps and streams in the local dark matter distribution, Nature (2008), [0805.1244v2].

[5] J. Coronado-Blázquez, M. A. Sánchez-Conde, A. Domínguez, A. Aguirre-Santaella, M. D. Mauro, N. Mirabal et al., Unidentified gamma-ray sources as targets for indirect dark matter detection with the Fermi-Large Area Telescope, JCAP 2019 (jul, 2019) 020-020.

[6] J. Coronado-Blázquez, M. A. Sánchez-Conde, M. D. Mauro, A. Aguirre-Santaella, I. Ciucă, A. Domínguez et al., Spectral and spatial analysis of the dark matter subhalo candidates among Fermi Large Area Telescope unidentified sources, JCAP 2019 (nov, 2019) 045-045.

[7] The MAGIC Collaboration, The major upgrade of the MAGIC telescopes, Part II: A performance study using observations of the Crab Nebula, Astroparticle Physics 72 (jan, 2016) 76-94.

[8] J. Knödlseder, M. Mayer, C. Deil, J.-B. Cayrou, E. Owen, N. Kelley-Hoskins et al., GammaLib and ctools, A\&A $\mathbf{5 9 3}$ (aug, 2016) A1.

[9] M. Cirelli, G. Corcella, A. Hektor, G. Hütsi, M. Kadastik, P. Panci et al., PPPC 4 DM ID: A Poor Particle Physicist Cookbook for Dark Matter Indirect Detection, JCAP (2010), [1012.4515v4].

[10] J. Conrad, Statistical issues in astrophysical searches for particle dark matter, Astroparticle Physics 62 (mar, 2015) 165-177.

[11] T. Sawala, C. S. Frenk, A. Fattahi, J. F. Navarro, T. Theuns, R. G. Bower et al., The chosen few: the low-mass haloes that host faint galaxies, MNRAS 456 (dec, 2015) 85-97. 